\title{
Desempenho inicial de mamoeiro em diversos substratos
}

\author{
Francisco Cleilson Lopes COSTA'; Antônia Thalyta Lopes SILVEIRA'; Felipe Hermínio Meireles NOGUEIRA'; \\ Allana Rayra Holanda SOTERO'; Maria Vanessa da Costa de DEUS; Cleilson do Nascimento UCHÔA ${ }^{l}$ \\ 凶llsncosta@gmail.com
}

1. Instituto Federal do Ceará, Rua Estevão Remigio, 1145, Ceará, Brasil.

Histórico do Artigo:

Recebido: 23 de abril de 2016

Aceito: 26 de outubro de 2016

Publicado: 22 de dezembro de 2016

\begin{abstract}
Resumo: A cultura do mamoeiro é uma das mais importantes no Brasil inteiro dos pontos de vista econômico e social, dada sua fácil adaptação aos diversos tipos de clima e solo. Assim sendo, conhecer a melhor forma de instalação desta cultura é um fator importante para subsidiar a melhor produção agrícola. 0 objetivo deste trabalho foi avaliar o desempenho de sementes de mamoeiro em substratos variados. 0 experimento foi realizado em casa de vegetação, localizada na Unidade Experimental de Pesquisa e Extensão do Instituto Federal de Educação, Ciência e Tecnologia do Ceará, Limoeiro do Norte. 0 delineamento experimental foi em blocos casualizados, com quatro blocos De quatro tratamentos com oito repetições, totalizando 256 plantas. Foram calculados o índice de velocidade de germinação e a taxa de germinação aos 13 e 20 dias após o plantio. Ao final, foi contabilizado o número de folhas, a massa foliar e a massa das raízes. A análise de dados foi feita como auxílio do software Assistat, que foi utilizado para a análise de variância e diferenças entre médias pelo teste de Tukey a 5\% de probabilidade. Todos os dados apresentavam normalidade (Shapiro-Wilk) e nenhum dado apresentou diferença significativa para tratamento ou bloco. Os múltiplos substratos e blocos não influenciaram o desempenho das sementes. Observou-se, entretanto, que doses mais concentradas de nitrogênio tendem a interferir na germinação e desenvolvimento inicial de plântulas de mamoeiro.
\end{abstract}

Palavras-chave: Compost-Aid®, Cultivo protegido, Influência de substratos.

\section{Initial performance of papaya in varies substrates}

\begin{abstract}
The papaya crop is one of the most important crops all over Brazil in the economic and social aspects, given its easy adaptation to different types of climate and soil. Therefore, to know the best means to install this cultivation is an important factor to improve agricultural production. The aim of this research was to assess papaya seeds' performance on several substrates. The experiment was carried out in a greenhouse located at the Experimental Unit for Research and Extension - UEPE - of the Federal Institute of Education, Science and Technology of Ceara - IFCE, Limoeiro do Norte, Ceará State, Brazil. The experimental design was randomized blocks with four blocks of four treatments and eight repetitions, totaling 256 plants. The speed of germination index and germination rate was calculated at 13 and 20 days after sowing. In the end, it was counted the number of leaves, leaves and roots mass. Data analysis was done through the Assistat software, which was used to verify analysis of variance and average differences by Tukey's test at 5\% probability. All data presented normality (Shapiro-Wilk) and no data has shown significant difference for treatment or block. Multiple substrates and blocks did not affect seeds' performance. However, it was noticed that higher levels of nitrogen tend to interfere with germination and early development of papaya seedlings.
\end{abstract}

Keywords: Compost-Aid®, Protected growth, Substrate influence. 


\title{
Desarrollo inicial de papaya en muchos sustratos
}

\begin{abstract}
Resumen: El cultivo de la papaya es uno de los más importantes en el Brasil en términos económicos y sociales, por su fácil adaptación a diferentes tipos de clima y solo. Entónces, conocer la mejor manera de instalar esta cultura es un factor importante para apoyar la producción agrícola. El objetivo de este estudio fue evaluar el rendimiento de semillas de papaya en sustratos variados. La investigación se llevó a cabo en un invernadero ubicado en la Unidade Experimental de Pesquisa e Extenção do Instituto Federal de Educação, Ciência e Tecnologia do Ceará, Campus Limoeiro do Norte, Estado do Ceará, Brasil. El diseño experimental fue bloques al azar con cuatro bloques de cuatro tratamientos y con ocho repeticiones, con un total de 256 plantas. Foram calculada la velocidad de germinación y el índice de velocidad de germinación a los 13 y 20 días después del plantio. Al final, fue contado la quantidad de hojas, la masa de hojas y la masa de raízes. El análisis de datos se hizo com ayuda de software Assistat, para el análisis de varianza y las diferencias entre las medias mediante la prueba de Tukey al $5 \%$ de probabilidade. Todos los datos mostraron normalidad (Shapiro-Wilk) y ninguno mostró diferencia significativa para los tratamientos o blocos. Los múltiples sustratos y bloques no afectaron al rendimiento de las semillas. Fue observado, sin embargo, que las dosis más concentradas de nitrógeno tienden a interferir con el desarrollo inicial de la germinación de semillas de papaya.

Palabras clave: Compost-Aid®, Cultivo protegido, Influencia de sustratos.
\end{abstract}

\section{INTRODUÇÃo}

No setor da Fruticultura, a cultura do mamoeiro é de grande representatividade, segundo dados de IBGE (2013) com 32.139 ha de área plantada ou destinada à colheita, 31.989 ha de área colhida, 1.582.638 t de produto colhido, com produtividade de 49.474 t.ha $^{-1}$, com valor da produção de R\$1.209.777.000,00, o que representa um aumento de 4,3\% em relação à produção ao ano anterior.

A adubação nitrogenada via adubos orgânicos apresenta-se como uma alternativa para a agricultura, além de aumentar a ação de microrganismos do solo que por sua vez disponibilizam os nutrientes à medida que a demanda por nutrientes na superfície de troca aumenta, melhorando sobremaneira as condições químicas, físicas e biológicas do solo.

Diversos tipos de materiais orgânicos podem ser adicionados ao solo com finalidade de melhorar suas condições: torta de mamona, cama de frango, esterco bovino, suíno, ovino, vinhaça etc. Em comparação com o esterco bovino, em termos de nitrogênio (N), 3,1\%, a vinhaça apresenta em torno de $0,5 \%$ de N. No entanto, materiais ricos em nitrogênio têm tendência de interferir no desenvolvimento inicial de plantas. Em trabalho com aplicação de vinhaça, dentre outros produtos, em girassol, mamona e amendoim, apresentou interferência sobre as características índice de velocidade de emergência, emergência final, primeira contagem de 
emergência, comprimento da parte aérea de plantas e biomassa seca da parte aérea de girassol e amendoim (Ramos et al. 2008).

Em trabalho com mamona, Oliveira et al. (2009), observaram que o lodo de esgoto corrigido apresentou melhor desempenho e dois tipos de solos tratados sem lodo apresentaram menor volume de raiz. Produtos industrializados vêm sendo muito utilizados para aumentar a eficiência da produção, de maneira geral, como Compost-Aid® que é um inoculante utilizado para aumentar a eficiência de liberação de nutrientes de adubos orgânicos, Plantmax® que é um substrato industrializado elaborado a partir de vermiculita expandida e materiais orgânicos vegetais livres de patógenos e dissemínulos de plantas invasoras (WAGNER JR. et al., 2006).

Trabalhando com níveis de sombreamento com plantas de Theobroma grandiflorum (Willd. Ex Spreng.), Silva et al. (2007) encontraram para recomendação de sombreamento em início de desenvolvimento, por apresentar melhor desempenho de alocação de massa a parte aérea, altura, diâmetro e número de folhas.

0 objetivo deste trabalho foi estudar os efeitos de Compost-Aid® e de diferentes tratamentos no desenvolvimento inicial de plantas de mamão.

\section{MATERIAL E MÉTODOS}

0 experimento realizado em casa de vegetação, na Unidade Experimental de Pesquisa e Extensão do Instituto Federal de Educação, Ciência e Tecnologia do Ceará, Limoeiro do Norte, na Chapada do Apodi, com coordenadas 5 $5^{\circ} 10^{\prime}$ latitude sul, $38^{\circ} 00^{\prime}$ de longitude oeste, altitude de 145,9 m. Conforme classificação de Köppen, o clima da região é BSw'h', ou seja, seco e muito quente, com regime pluviométrico anual de $822,4 \mathrm{~mm}$. Com duas estações climáticas: seca que vai de junho a janeiro, e chuvosa, de fevereiro a maio, normalmente.

0 delineamento experimental foi blocos casualizados, com quatro blocos e quatro tratamentos com 16 repetições, totalizando 256 plantas na área útil. Foram montados quatro blocos equivalendo a concentrações do produto Compost-Aid ${ }^{\circledR}$, um enriquecedor para a matéria orgânica do solo: B1 = 0 g. $\mathrm{Mg}^{-1}$; B2 = 100 g. $\mathrm{Mg}^{-1} ; \mathrm{B} 3=150$ g. $\mathrm{Mg}^{-1}$; B4 = 200 g. $\mathrm{Mg}^{-1}$. 0s tratamentos foram compostos por variação na percentagem dos componentes: $\mathrm{Tl}=(1 / 3$ areia $+1 / 3$ esterco + 1/3 casca de côco $) ; \mathrm{T} 2$ = (1/2 areia + 1/4 esterco + 1/4 casca de côco); T3 = $(1 / 4$ areia + 1/2 esterco $+1 / 4$ casca de côco); $\mathrm{T} 4$ = (1/4 areia + 1/4 esterco + 1/2 casca de côco). 0s componentes utilizados 
foram areia de formigueiro, pela sua riqueza em nutrientes minerais e compostos orgânicos e sua estilidade; esterco curtido de curral e casca de côco seca e moída.

0s substratos foram peneirados, pesados em balança de precisão semi analítica, com uma casa decimal e misturados. Depois de misturados os componentes, foi separada uma quantidade para cada tratamento, utilizando um recipiente de volume conhecido (1 L), aproximadamente $800 \mathrm{~g}$, e utilizando esse valor para a obter a massa equivalente às doses de Compost-Aid® dos blocos: Bl = 0 mg; B2 = 80 mg; B3 = 120 mg; B4 = 160 mg. Para pesagem das doses de Compost-Aid@ foi utilizada balança analítica de precisão com quatro casas decimais.

Depois de os substratos, as bandejas para germinação com capacidade de 50 sementes (10x5) foram preenchidas, regadas e as sementes foram semeadas na quantidade de duas sementes de uma determinada marca comercial por célula para posterior desbaste daquela menos vigorosa.

Para o cálculo do índice de velocidade de germinação (IVG), sugerido por Maguire (1962) aos 20 dias após o plantio, foi utilizada a fórmula (1):

$$
\sum_{i=1}^{n} I V G=\frac{N S G_{1}}{N T S}+\frac{N S G_{2}}{N T S}+\cdots+\frac{N S G_{n}}{N T S}
$$

Em que, $\Sigma$ IVG: somatório dos índices de velocidade de germinação, no i:iésimo até o n: enésimo dia; NSG: número de sementes germinadas no dia 1 até dia n; NTS: número total de sementes por tratamento.

A taxa de germinação média (\%) foi calculada aos 13 e 20 dias após o plantio, pela fórmula (2):

$$
\% T G=\left\{\frac{\left[\left(\frac{N S G_{13} \mathrm{o} d i a}{N T S}\right) \times 100\right]+\left[\left(\frac{N S G_{20 \mathrm{o}} \mathrm{dia}}{N T S}\right) \times 100\right]}{2}\right\}
$$

Ao final, foi contabilizado o número de folhas, a massa fresca foliar (g) e a massa fresca das raízes (g), utilizando balança analítica de precisão com quatro casas decimais. Para cálculo da massa fresca média, foi utilizada a fórmula (3):

$$
M F=\frac{\text { massa das folhas }(g)}{\text { número de folhas }}
$$




\section{RESULTADOS E DISCUSSÃ0}

A tabela 1 apresenta os dados de análise de variância da taxa de germinação e do índice de velocidade de germinação, mostrando que nenhum dos tratamentos ou blocos apresentou diferença significativa. A taxa média de germinação foi 68,16\%. 0 índice médio de velocidade de germinação foi de 1,36.

Tabela 1 - Análise de variância da taxa de germinação, índice de velocidade de germinação

\begin{tabular}{lccccc}
\hline \multirow{1}{*}{ ANAVA } & \multicolumn{2}{c}{ TAXA DE GERMINAÇ̃̃o } & \multicolumn{1}{c}{ ÍNDICE DE VELOCIDADE DE GERMINAÇÃ0 } \\
& QM & F & QM & F \\
\hline Bloco & 57,58 & $0,56^{\mathrm{ns}}$ & 0,063 & & $1,53^{\mathrm{ns}}$ \\
Tratamento & 156,86 & $1,53^{\mathrm{ns}}$ & 0,023 & & $0,56^{\mathrm{ns}}$ \\
Média & \multicolumn{2}{c}{68,16} & & 1,36 & \\
\% Coef. Variação & \multicolumn{2}{r}{14,86} & & 14,86 & \\
\hline
\end{tabular}

Fonte: 0 s autores.

0 percentual de germinação mínimo esperado foi de 75\% o que pode ser justificado pelo baixo vigor ou potencial genético da cultivar em estudo. Pode ter ocorrido ataque de formigas cortadeiras, que é comum na área de realização do estudo, o que pode ter reduzido o número de plantas na contagem. No entanto, não foi observado nenhum ataque, nem folhas cortadas ou repicadas no solo ou bandeja.

A tabela 2 apresenta os dados de análise de variância para o número médio de folhas, a massa fresca média de folhas, a massa fresca média de raízes e a relação entre massa de raízes e massa foliar. 0 número médio de folhas foi de 3,55. A massa fresca média das folhas (g) foi $0,063(\mathrm{~g})$, a massa fresca média de raiz foi de $0,105(\mathrm{~g})$ e a razão entre massa fresca de raízes e massa fresca média de folhas foi 1,66.

Tabela 2 - Análise de variância de atributos do mamoeiro

\begin{tabular}{lcccccccccc}
\hline \multirow{2}{*}{ ANAVA } & \multicolumn{2}{c}{ NF } & \multicolumn{2}{c}{ MF } & \multicolumn{2}{c}{ MR } & \multicolumn{2}{c}{ MR/MF } & \multicolumn{2}{c}{ MF/MR } \\
& QM & F & QM & F & QM & F & QM & F & QM & F \\
\hline Bloco & 0,066 & $0,61^{\mathrm{ns}}$ & 0,00003 & $0,09^{\mathrm{ns}}$ & 0,0003 & $0,27^{\mathrm{ns}}$ & 0,036 & $0,23^{\mathrm{ns}}$ & 0,0069 & $0,290^{\mathrm{ns}}$ \\
Tratamento & 0,41 & $3,79^{\mathrm{ns}}$ & 0,00054 & $1,76^{\mathrm{ns}}$ & 0,0034 & $2,74^{\mathrm{ns}}$ & 0,19 & $1,21^{\mathrm{ns}}$ & 0,0388 & $1.628^{\mathrm{ns}}$ \\
Média & 3,55 & 0,063 & 0,105 & 1,66 & 0,632 \\
CV\% & 9,27 & & 27,78 & & 33,33 & 23,56 & 24,4 \\
\hline
\end{tabular}

Legenda: NF: número de folhas; MF: massa foliar; MR: massa de raiz; MR/MF: relação entre massa de raiz e massa foliar. 
Fonte: 0s autores.

Esperava-se que um efeito significativo fosse observado no tratamento T3B3 ou T3B4, já que apresentam maiores níveis de esterco e de Compost-Aid®, já que o inoculante que tem a função de acelerar a compostagem, causando um abaixamento na relação $\mathrm{C} / \mathrm{N}$ do substrato. 0 esterco tem alto teor de nitrogênio (N) apresentando uma composição média de 3,1\% (UFLA, 2014). A função esperada para o Compost-Aid® nesta situação seria liberar o N, provocando um diferencial no crescimento das raízes e da parte aérea. No entanto, nenhuma das características apresentadas na tabela 2 apresentou diferença significativa.

A relação entre massa de raiz e parte aérea $(1,66)$ indica que nesse primeiro momento de crescimento, as raízes apresentaram maior massa, podendo nos próximos estádios passar a ser inferior a um, quando a parte aérea representar maior parte. Pode-se sugerir uma variação no sombreamento para permitir possível variação na massa da parte aérea. Observando-se os estudos de Silva et al. (2007), 50\% de sombreamento proporcionou a melhor condição para utilização eficiente da incidência solar, em comparação com 0\% e 90\% de sombreamento em plantas de Theobroma grandiflorum. Possivelmente, um sobreamento diferenciado proporcionará condições mais adequadas para o melhor desenvolvimento de plantas de mamoeiro.

Certa variabilidade genética das sementes ocorreu, onde algumas crescem e acumulam mais massa que outras, já que a condição ambiente não apresentou diferença, o que é enfatizado pelo Coeficiente de Variação (\%CV) = 23,56\%, o que corroborou com os \%CV das massas das folhas e massas das raízes, que ficaram em torno de 30\%, já se aproximando do alto grau de variação.

A tabela 3 apresenta equações de regressão e respectivos coeficientes de determinação para interação entre características e doses de Compost-Aid® e esterco.

Todas as características em interação com Compost-Aid® apresentaram coeficiente de determinação acima de $90 \%$, exceto o percentual de germinação que ficou em torno de $62 \%$. Pela análise de variância, nenhum teste apresentou significância, mas ao se obter dispersão dessas características, observou-se que essas características apresentam comportamento relacionado e crescente. 
Tabela 3 - Dispersão das características analisadas e blocos e tratamentos

\begin{tabular}{|c|c|c|}
\hline INTERAÇÃ0 & COMPOST-AID & ESTERC0 \\
\hline TX GERM & $\begin{array}{c}y=-0,0005 x^{2}+0,1468 x+60,788 \\
R^{2}=0,6264\end{array}$ & $\begin{array}{c}\mathrm{y}=4,6881 \mathrm{x}^{2}-45,942 \mathrm{x}+170,41 \\
\mathrm{R}^{2}=0,9767\end{array}$ \\
\hline IVG & $\begin{array}{c}\mathrm{y}=-1 \mathrm{E}-05 \mathrm{x}^{2}+0,0025 \mathrm{x}+1,3422 \\
\mathrm{R}^{2}=0,9845\end{array}$ & $\begin{array}{c}y=0,0119 x^{2}-0,0942 x+1,529 \\
R^{2}=0,1449\end{array}$ \\
\hline $\mathrm{NF}$ & $\begin{array}{c}y=-5 E-05 x^{2}+0,012 x+3,1733 \\
R^{2}=0,9278\end{array}$ & $\begin{array}{c}y=0,2147 x^{2}-1,9081 x+7,4146 \\
R^{2}=0,7714\end{array}$ \\
\hline MF & $\begin{array}{c}y=-2 E-06 x^{2}+0,0004 x+0,0516 \\
R^{2}=0,8932\end{array}$ & $\begin{array}{c}y=0,0073 x^{2}-0,0689 x+0,2121 \\
R^{2}=0,5096\end{array}$ \\
\hline MR & $\begin{array}{c}y=-5 E-06 x^{2}+0,001 x+0,0896 \\
R^{2}=0,9357\end{array}$ & $\begin{array}{c}y=0,0101 x^{2}-0,0945 x+0,308 \\
R^{2}=0,1529\end{array}$ \\
\hline $\mathrm{MR} / \mathrm{MF}$ & $\begin{array}{c}y=-3 E-05 x^{2}+0,0035 x+1,7764 \\
R^{2}=0,9963\end{array}$ & $\begin{array}{c}y=-0,0674 x^{2}+0,6611 x+0,1912 \\
R^{2}=0,1715\end{array}$ \\
\hline $\mathrm{MF} / \mathrm{MR}$ & $\begin{array}{c}y=1 E-05 x^{2}-0,0015 x+0,5777 \\
R^{2}=0,9991\end{array}$ & $\begin{array}{c}\mathrm{y}=0,0318 \mathrm{x}^{2}-0,3055 \mathrm{x}+1,2999 \\
\mathrm{R}^{2}=0,1487\end{array}$ \\
\hline
\end{tabular}

Fonte: 0 s autores.

Todas as características em interação com Compost-Aid ${ }^{\circledR}$ apresentaram coeficiente de determinação acima de $90 \%$, exceto o percentual de germinação que ficou em torno de $62 \%$. Pela análise de variância, nenhum teste apresentou significância, mas ao se obter dispersão dessas características, observou-se que essas características apresentam comportamento relacionado e crescente.

Para as interações com esterco, índice de velocidade de germinação, massa de raízes e razão entre massa de raízes e massa foliar, apresentaram coeficiente de determinação em torno de 15\%, considerado baixo, com tendência a apresentarem efeitos contrários. Estudos sobre este fato devem ser realizados de maneira mais específica, com aumento das doses de esterco sobre o desenvolvimento de plantas. Parece que o efeito contrário do esterco está envolvido na decomposição de materiais orgânicos pelos microrganismos do solo que causam perda de $\mathrm{C}-\mathrm{CO}_{2}$ e liberam subprodutos, que por serem mais recalcitrantes, levam a esses microrganismos específicos a absorverem mais carbono para crescimento e podendo haver competição com as plantas em desenvolvimento. É um caso que merece estudo mais detalhado (UFLA, 2014).

Do mesmo modo, a interação entre taxa de germinação e esterco apresentou coeficiente de determinação de aproximadamente 98\%. Ramos et al. (2008) observaram que a vinhaça apresentou interferência sobre a emergência de girassol e amendoim. Wagner Jr. et al. (2006) 
perceberam que substratos de boa indicação para desenvolvimento inicial de plantas de maracujazeiro amarelo foram Plantmax $®$, Plantmax $®$ + areia (l:1 v/v), Plantmax® + Latossolo Vermelho (l:1 v/v), Plantmax® + Latossolo Vermelho + areia (1:1:1 v/v).

\section{CONCLUSÃ0}

As doses de Compost-Aid® e tipos de substratos não interferiram no desenvolvimento inicial de plantas de mamão e em maiores concentrações de adubos orgânicos nitrogenados houve tendência de interferência no desenvolvimento inicial de plantas. Outros produtos em substituição do Compost-Aid® são mais efetivos na promoção do desenvolvimento inicial de plantas, sendo este produto mais indicado para tratamento de substratos nas etapas anteriores a semeadura.

\section{REFERÊNCIAS BIBLIOGRÁFICAS}

IBGE. Produção agrícola municipal: culturas temporárias e permanentes: Brasil 2013. Rio de Janeiro: IBGE, 2013.

MAGUIRE, J.D. Speed of germination aid in selection and evaluation for seedling and vigor. Crop Science, Madison, v.2, n.2, p.176-177, 1962.

OLIVEIRA, J. P. B.; LOPES, J. C.; ALEXANDRE, R. S.; JASPER, A. P. S.; SANTOS, L. N. S.; OLIVEIRA, L. B. Efeito do lodo de esgoto no desenvolvimento inicial de duas cultivares de mamona em dois tipos de solos. Engenharia Ambiental, Espírito Santo do Pinhal, v.6, n.2, p. 174-180, 2009.

PAIVA, R.; SOUZA, B.D.; PEREIRA, J.; CARVALHO, F.D.M.; CURI, N. [Eds]. Fertilizantes orgânicos: usos, legislação e métodos de análise. Lavras/MG. Boletim Técnico. n.96, p.1-90, 2014.

RAMOS, N. P.; NOVO, M. D0 C. DE S. S.; UNGAR0, M. R. G.; DO LAG0, A. A.; MARIN, G. C. Efeito da vinhaça no desenvolvimento inicial de girassol, mamona e amendoim em casa de vegetação. Bragantia, Campinas, v.67, n.3, p.685-692, 2008.

SILVA, R.R.; FREITAS, G.A.; SIEBENEICHLER, S.C.; MATA, J.F.; CHAGAS, J.R. Desenvolvimento inicial de Theobroma grandiflorum (Willd. Ex Spreng.) Schum. Sob influência de sombreamento. Acta Amazônica, Manaus, v.37, n.2, p.365370, 2007.

UFLA. Fertilizantes orgânicos: usos, legislação e métodos de análise. Boletim Técnico. Lavras. n.96, p.1-90, 2014.

WAGNER JR., A.; ALEXANDRE, R.S.; NEGREIROS, J.R.S.; PIMENTELL.D.; SILVA, J.O.C.; BRUCKNER, C.H. Influência do substrato na gemrinação e desenvolvimento inicial de plantas de maracujazeiro amarelo (Passiflora edulis Sims f. flavicarpa Deg). Ciência Agrotécnica, Lavras, v. 30, n. 4, p. 643-647, 2006. 